\title{
Numerical simulations of cyclic voltammetry for lithium-ion intercalation in nanosized systems: finiteness of diffusion versus electrode kinetics
}

\author{
E.M. Gavilán-Arriazu ${ }^{1,2}$ - M.P. Mercer ${ }^{3,4,5}$ • O.A. Pinto ${ }^{2}$ • O.A. Oviedo ${ }^{1}$ • D.E. Barraco ${ }^{6}$ - H.E. Hoster ${ }^{3,4,5}$ • E.P.M. Leiva ${ }^{1}$ (iD
}

Received: 30 April 2020 / Revised: 5 June 2020 / Accepted: 7 June 2020

(C) Springer-Verlag GmbH Germany, part of Springer Nature 2020

\begin{abstract}
The voltammetric behavior of $\mathrm{Li}^{+}$intercalation/deintercalation in/from $\mathrm{LiMn}_{2} \mathrm{O}_{4}$ thin films and single particles is simulated, supporting very recent experimental results. Experiments and calculations both show that particle size and geometry are crucial for the electrochemical response. A remarkable outcome of this research is that higher potential sweep rates, of the order of several millivolts per second, may be used to characterize nanoparticles by voltammetry sweeps, as compared with macroscopic systems. This is in line with previous conclusions drawn for related single particle systems using kinetic Monte Carlo simulations. The impact of electrode kinetics and finite space diffusion on the reversibility of the process and the finiteness of the diffusion in ion $\mathrm{Li} / \mathrm{LiMn}_{2} \mathrm{O}_{4}$ (de)intercalation is also discussed in terms of preexisting modeling.
\end{abstract}

\section{Introduction}

Lithium-ion batteries are widely used in small electronic devices and in the automotive industry. Proper design of the materials is a crucial stage for the operation of these types of batteries. In this sense, mathematical models play an important role. To simulate numerical cyclic voltammetry profiles for (de)intercalation of $\mathrm{Li}^{+}$in $\mathrm{LiMn}_{2} \mathrm{O}_{4}$ and $\mathrm{LiCoO}_{2}$ cathodes, Vassiliev et al. [1] have designed a selfconsistent mathematical model, not only suitable for reproducing experimental data, but for predicting kinetic, thermodynamic and transport parameters. This model was compared and fitted with multi-particle (bulk) experiments using $\mathrm{LiMn}_{2} \mathrm{O}_{4}$ and $\mathrm{LiCoO}_{2}$ cathodes in organic solvents and is used here, in the present work, to analyze the behavior of nanosystems. Planar and spherical geometries are approximations generally used to mimic lithium manganese oxide (LMO) thin films, porous electrodes and single particles $[2,3]$, which are the most common types of electrode material configuration [4-11]. LMO nanorods, which can be compared with a cylindrical geometry, have also been used $[12,13]$. Furthermore, $\mathrm{LiMn}_{2} \mathrm{O}_{4}$ has the potential to be recycled in a simple way, and reused in layered form as cathode material for Sodium ion batteries [14].

This article is dedicated to Prof. Fritz Scholz on the occasion of his $65^{\text {th }}$ birthday. Es ist ein Vergnügen, mit so einem Chefredakteur zusammenzuarbeiten.

E.M. Gavilán-Arriazu maxigavilan@hotmail.com

E.P.M. Leiva

eze_leiva@yahoo.com.ar

1 Departamento de Química Teórica y Computacional, Facultad de Ciencias Químicas, Universidad Nacional de Córdoba, INFIQC, Córdoba, Argentina

2 Instituto de Bionanotecnología del NOA (INBIONATEC), Universidad Nacional de Santiago del Estero (UNSE), G4206XCP Santiago del Estero, Argentina
3 Department of Chemistry, Lancaster University, Bailrigg, Lancaster, UK

4 ALISTORE European Research Institute CNRS FR 3104, Hub de l'Energie, 80039 Amiens, France

5 The Faraday Institution, Harwell Science and Innovation Campus, Didcot, UK

6 Facultad de Matemática, Astronomía y Física, IFEG-CONICET, Universidad Nacional de Córdoba, Córdoba, Argentina 
Very recently, Tao et al. [15] have performed for the first time experimental voltammograms for $\mathrm{LiMn}_{2} \mathrm{O}_{4}$ (LMO) nanometric-sized individual particles of known geometry and nanostructure, using scanning electrochemical cell microscopy (SECCM). They found that structural and geometric properties of the particles impact on the electrochemical response, allowing characterization by sweep rates 2-4 orders of magnitude higher than those used with multi-particle systems (1 V.s ${ }^{-1}$ vs $0.1-10 \mathrm{mV} . \mathrm{s}^{-1}$ ). Voltammograms were obtained for different individual $\mathrm{LiMn}_{2} \mathrm{O}_{4}$ particles, of about $200 \mathrm{~nm}$ in diameter, supported on glassy carbon (GC), in aqueous media. Another recent work is that of Mürter et al. [16], who analyzed the electrochemistry of LMO nanofilms with thicknesses ranging from $55 \mathrm{~nm}$ to $515 \mathrm{~nm}$, composed of nanocrystals in organic solvents, and showed how film thickness determines the behavior of voltammograms. These are valuable steps for the rational design of electrode materials for Li-ion batteries.

Nanosize effects in lithium ion insertion/deinsertion are also expected for other systems. For example, we found in kinetic Monte Carlo (kMC) simulations of graphite nanoparticles that well resolved voltammetric features were obtained at sweep rates several orders of magnitude higher than those used in multi-particle experimental systems [17, 18]. The model proposed in these articles was suitable for describing other relevant characteristics of Li-ion/graphite system like the criticality of phase transition [19-22], the intercalation in the equilibrium $[23,24]$ and the influence on kinetics on Li intercalation [25].

Concerning the theory of voltammetry under different regimes, it is also worth mentioning the contributions of Aoki et al. [26, 27], which provide a theoretical framework for analyzing the voltammetric profiles for finite diffusion space films. This model describes kinetic and finite diffusion effects in terms of two dimensionless parameters, namely $\Lambda$ and $w$. Calculated voltammograms were used to build different domains in a $\log (\Lambda)$ vs $\log (w)$ representation, where different regions were identified in terms of the reversibility, $\Lambda$, and the extent of the diffusion phenomena, $w$. This representation allows a straightforward prediction of the behavior of different systems in terms of experimentally measurable parameters. In this respect, Hjelm et al. [2] have used the same semi-infinite diffusion domains from Aoki et al. to predict the kinetic parameters for LMO, using convolution voltammetry. Recently, the coupling of electrochemistry with transport in other electrochemical techniques, such as AC voltammetry has also been demonstrated [28].

The purpose of this work is to use numerical simulations to obtain theoretical voltammograms for $\mathrm{LiMn}_{2} \mathrm{O}_{4}$ single particles and thin films of nanometric size in connection with the experimental works of Tao et al. [15] and Mürter et al. [16], and analyze the relevance of the nanometric size of the particles for the voltammetric response of the system. The results will be also discussed in the framework of the theoretical predictions of Aoki et al. [27].

In the present article we will show that electrode structure and geometry at the nanometric level may have an influence on the charging/discharging process of the material. Higher charging/discharging rates and a high grade of reversibility are expected for nanometric sized particles, since diffusion control is minimized.

While section 2 presents the mathematical model used for simulating numerical voltammograms, section 3 shows the results and discussion and section 4 summarizes the most relevant conclusions of the present work.

\section{Mathematical model}

The theoretical model for simulating cyclic voltammograms used here was adopted from Vassiliev et al. [1], and consists in the numerical resolution of Fick's diffusion equation, equation (1), using the Crank-Nicolson method [1, 3, 29].

$\frac{\partial \theta}{\partial t}=D\left(\frac{\partial^{2} \theta}{\partial x^{2}}+\frac{z}{x} \frac{\partial \theta}{\partial x}\right)$

Here $\theta$ is the $\mathrm{Li}^{+}$occupation fraction inside the electrode, $x$ is the distance measured from the electrode center $(x=0)$ towards the surface of the particle $(x=h), D$ is the diffusion coefficient and $z$ is a number given by the geometry of the diffusion problem: $z=0$ planar, $z=1$ cylindrical and $z=2$ spherical, according to the formalisms developed in references $[30,31]$.

The model assumes a Frumkin-based intercalation isotherm [32] with several interaction terms, and considers the equilibrium potential of the two phases participating independently [1]. The equilibrium potential $E(\theta)^{e q}$ is given by:

$$
\begin{aligned}
& E(\theta)^{e q}=\frac{R T}{F} \ln \left(\frac{1-\theta}{\theta}\right) \\
& +\left\{E_{1 / 2}+\frac{R T}{F}\left[g_{1}(0.5-\theta)+g_{2}(0.5-\theta)^{2}+g_{3}(0.5-\theta)^{3}\right]\right\}
\end{aligned}
$$

where $R$ is the gas constant, $T$ is the absolute temperature, $F$ is the Faraday constant, $E_{1 / 2}$ is the standard redox potential (the equilibrium potential of a phase having $\theta=0.5$ measured versus a metallic Li reference electrode) and $g_{1}, g_{2}, g_{3}$ are dimensionless interaction parameters. The term inside the keys is denoted with $E_{0}(\theta)$. This is a simplification of the problem, which involves an order-disorder transition [33, 34]. In particular, the first term of equation 2 describes the configurational entropy of an ideal solid solution, which is 
not a completely correct description at close to $\theta=0.5$ due to ordering of the lattice sites. However, we adopted the approximation to consistently compare with the results of Vassiliev et al. and to reduce the computational complexity of the model. We did not use the classical Frumkin isotherm because that kind of approximation (with only a single "global" interaction) was found by Vassiliev et al. [1] as too simple to reproduce the energetics of Li-ion intercalation in LMO. Equation (2) provides a greater flexibility and involves a Taylor expansion, around $\theta=0.5$, of the free energy of insertion up to third order.

For the voltammetric current obtained at a given applied potential, the Butler-Volmer equation is used:

$$
\begin{aligned}
& I(E)=S F \frac{\rho . n_{L i}}{M_{r}} k_{s} \\
& \left\{\theta \exp \left[\frac{(1-\alpha) F\left(E-E_{0}(\theta)\right)}{R T}\right]-(1-\theta) \exp \left[\frac{\alpha F\left(E-E_{0}(\theta)\right)}{R T}\right]\right\}
\end{aligned}
$$

where $\rho$ is the phase density, $n_{L i}$ the number of intercalating $\mathrm{Li}^{+}, M_{r}$ the molecular mass, $k_{s}$ the heterogeneous rate constant, $\alpha$ the transfer coefficient and $E$ the electrode potential. $\mathrm{S}$, the electrode surface area, is given by

$S=\frac{j m}{\rho d}$,

$m$ being the mass of the intercalating electrode material and $d$ being the cross-section in the direction of $\mathrm{Li}^{+}$diffusion, such that $d=2 h$. For a spherical geometry it is more usual to talk about particle size (diameter) $d$, and for planar geometry of layer thickness $h$, but in both cases the diffusion length is defined by $h$. Finally, $j$ is a number that depends on the type of geometry, $j=2$ (planar), $j=4$ (cylindrical), $j=6$ (spherical). In the present work we have considered planar and spherical geometries.

The boundary conditions at the center of the particle $(x=0)$ and at the surface $(x=h)$ are:

$$
\begin{aligned}
& \left(\frac{\partial \theta}{\partial r}\right)_{0}=0 \\
& \left(\frac{\partial \theta}{\partial r}\right)_{h}=\frac{-I(E)}{S F D \frac{\rho \cdot n_{L i}}{M_{r}}}
\end{aligned}
$$

The initial condition imposed at $t=0$ is a homogeneous $\mathrm{Li}^{+}$ occupation for all $x$ : $\theta(x, 0)=\theta\left(E_{i}\right)$

where $\theta\left(E_{i}\right)$ is the equilibrium electrode occupation calculated with equation (2) at the initial potential $E_{i}$.

\section{Results and discussion}

\section{Global picture. Reversibility and finiteness of diffusion length}

Before starting with the description of the present numerical simulations, we will briefly analyze particle size and sweep rate effects in the light of the work of Aoki et al. [27]. These authors implemented a theoretical model to study voltammetry for finite diffusion space films. This kind of study should be applicable to intercalation systems, since, in principle, the phenomena are comparable. As discussed by Conway [35], concerning thermodynamics, the insertion of $\mathrm{Li}$ ions into layer-lattice host materials share a number of common features with the behavior of two-dimensional underpotential deposition (upd). The main difference between insertion and upd systems is given by the fact that ion diffusion plays an important role in the former, and this is a key feature in the modeling of reference [27].

As pointed out in the introduction, Aoki et al. [27] made a useful classification of the voltammetric behavior of a simple electrochemical reaction in a finite diffusion space using two dimensionless parameters. Using the present notation, these two parameters are:

$$
\begin{aligned}
& \Lambda=k_{S}(R T / n F D v)^{1 / 2} \\
& w=n F v h^{2} / R T D
\end{aligned}
$$

Concerning the reaction rate, the cases identified were those of reversible (1), quasi-reversible (2) and the totally irreversible waves (3), and concerning the diffusional behavior, the cases arising where those of semi-infinite diffusion (A), finite diffusion case (B) and surface waves (C). We have kept the original notation of Aoki et al. [27] for the different regimes, and the different situations are illustrated in Fig. 1.

It can be easily verified that equation (4) of Aoki et al. [27] is equivalent to equation ( 3 ) of the present work provided $g_{1}=$ $g_{2}=g_{3}=0$. Thus, it is interesting to use the kinetic parameter $\Lambda$ and the finite space diffusion parameter $w$, introduced in that work, to analyze the degree of reversibility and finiteness of the diffusion space for the experimental conditions used in reference [15]. This kind of plot is useful, since from the knowledge of the diffusional and kinetic parameters, one can predict the type of behavior expected.

$\mathrm{Li}$-ion intercalation/deintercalation into/from $\mathrm{LiMn}_{2} \mathrm{O}_{4}$ occurs in two steps, observed as two voltage plateaus in constant 


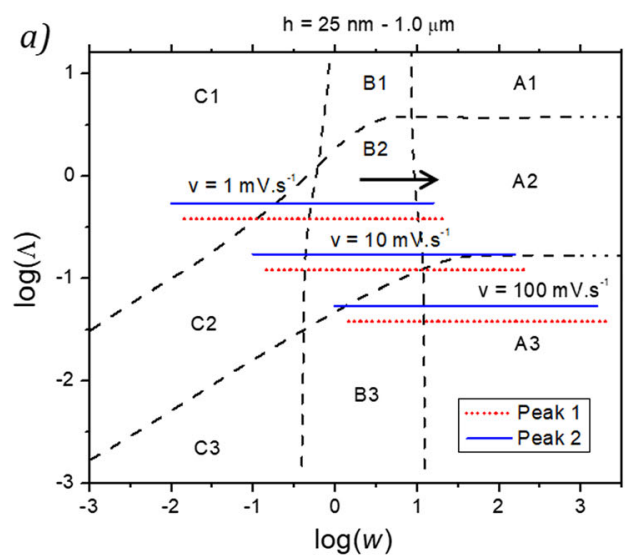

Fig. 1 Degree of reversibility, $\Lambda$ and finiteness of the diffusion $w$, for $\mathrm{Li}$ ion / LMO (de)intercalation, using the parameters $k_{s}$ and $D$ from reference [1] for peak 1 ( $L M O \leftrightarrow$ stage II transition) in red dotted line and peak 2 (stage $I I \leftrightarrow$ stage $I$ transition) in blue solid line. a) For $v=1 \mathrm{mV} . \mathrm{s}^{-1}$, $10 \mathrm{mV} \cdot \mathrm{s}^{-1}$ and $100 \mathrm{mV} . \mathrm{s}^{-1}$, varying the diffusion length from $25 \mathrm{~nm}$ to

current experiments and evident in two well defined peaks in cyclic voltammograms $[2,36]$. If we consider the first intercalation step from $4.3 \mathrm{~V}$ vs. $\mathrm{Li}$, the host lattice is initially empty of Li. Sweeping the potential negatively, half of the tetrahedral $8 \mathrm{a}$ sites of the crystal structure of the $\mathrm{LiMn}_{2} \mathrm{O}_{4}$ spinel ( $F d \overline{3} m$ space group) are occupied by $\mathrm{Li}$ ions to form the so-called stage II (first CV peak in the reductive sweep). The second step, at lower electrode potentials, consists in the filling of the other half of the 8a sites, to form stage I (second reductive $\mathrm{CV}$ peak). We denote the more positive one as peak 1 and the more negative one as peak 2. Peak 1 corresponds to the formation of the so-called stage II and peak 2 to the transition stage II $\leftrightarrow$ stage I [1]. Following the work of Aoki et al. [27], in the $\log (\Lambda)-\log (w)$ diagrams we consider the variation of $h$ for constant $v$ values (Fig. 1a) and the variation of the sweep rate $v$ for constant $h$ values (Fig. $1 b$ ). The $h$ and $v$ intervals are given at the top of the respective plots. The lines drawn there correspond to peak 1 (red dotted line) and to peak 2 (blue solid line). Since values of rate constants $k_{s}$ and diffusion coefficients $D$ must be assumed, we have adopted the values proposed by Vassiliev et al. [1].

From Figure $1 a$ we conclude that for $v=1 \mathrm{mV} \cdot \mathrm{s}^{-1}$ peaks 1 and 2 are in the surface waves and reversible region (C1) for the smallest sizes considered. For larger sizes there is a change to $\mathrm{C} 2$ and then to B2. Finally, for the largest particles, the lines are in the A2 domain. For $v=10 \mathrm{mV} . \mathrm{s}^{-1}$, peaks 1 and 2 are in the $\mathrm{C} 2$ region for the smallest sizes. For medium sizes, there is a change to the $\mathrm{B} 2$ domain and then for the largest particles peak 2 falls into the $\mathrm{A} 2$ domain and peak 1 in the $\mathrm{A} 3$ one. For a relatively large sweep rate, $v=100 \mathrm{mV} \cdot \mathrm{s}^{-1}$ both peaks move from $\mathrm{B} 3$ to $\mathrm{A} 3$ as particle size increases.

For a constant particle size and different sweep rates, a straight line with slope of $-1 / 2$ should follow in the $\log (\Lambda) v s \log (w)$ domain, as presented in Figure $1 b$. For $h=$ $134 \mathrm{~nm}$, the system crosses through different domains as

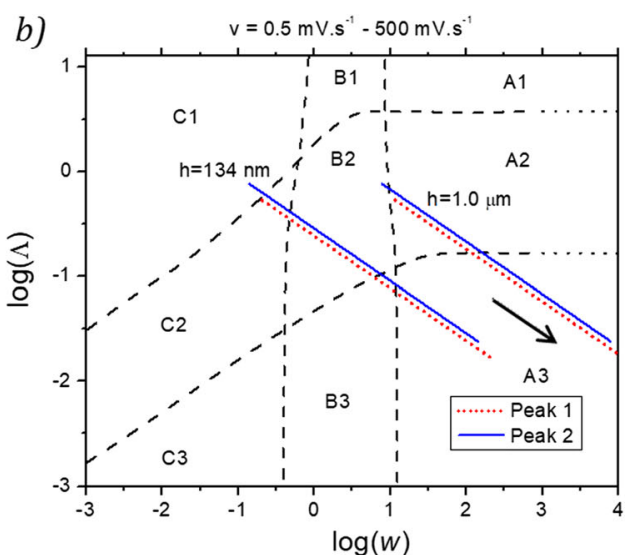

$1.0 \mu \mathrm{m} . b$ ) For constant $h=134 \mathrm{~nm}$ and $h=1 \mu \mathrm{m}$, varying the sweep rate from $0.5 \mathrm{mV} . \mathrm{s}^{-1}$ to $500 \mathrm{mV} \cdot \mathrm{s}^{-1}$. Black arrows show the direction of increasing size (Figure $a$ ) and increasing sweep rate (Figure $b$ ). The respective ranges of size and sweep rate are also indicated at the top of the figure

sweep rate rises: $\mathrm{C} 1, \mathrm{C} 2, \mathrm{~B} 2, \mathrm{~B} 3$ and $\mathrm{A} 3$. For $h=1 \mu \mathrm{m}$, peak 2 changes from $\mathrm{B} 2$, for the smallest size, to $\mathrm{A} 2$ and to $\mathrm{A} 3$ at $v$ rises; for the peak 1 there is only a change from $\mathrm{A} 2$ to $\mathrm{A} 3$ with $v$. Thus, only changes from the quasi-reversible to the irreversible regime are observed.

From the previous discussion, we find that the intercalation process should be strongly sensitive to the size of the lithiated particle. This fact must be taken into account for the formulation of proper models that allow understanding and predicting the system behavior for better material design.

\section{Size effect on cyclic voltammetry}

Now, we continue with the most relevant results from the present work. In this section, we will address the cyclic voltammetry of single $\mathrm{LiMn}_{2} \mathrm{O}_{4}$ particles and thin film crystals with numerical simulations, comparing our results with experimental data from Mürter et al. [16] and Tao et al. [15]. We have adopted the parameter values for LMO used by Vassiliev et al. [1], detailed in Table 1, which were fitted to experimental measurements with composite electrodes. In the following set of calculations, $m, S$ and $d$ were adapted to model single particles and thin films of different sizes. With this purpose, we have used different film thicknesses, comparable to those of Reference [16], and selected different particle volumes from the supplementary information of Tao et al. [15].

Figure 2 shows voltammograms for the case of planar diffusion, aimed to simulate thin film electrodes at different potential sweep rates. With visualization purposes, the simulations are separated in partial panels for $h=134 \mathrm{~nm}(a, b$ and $c)$, $h=300 \mathrm{~nm}(d, e$ and $f)$ and $h=1 \mu \mathrm{m}$ ( $g$ and $h$ ), and the current was normalized to the sweep rate. Sweep rates were selected such that a wide range of voltammetric responses can be observed, ranging from well-resolved current peaks to those where the voltammetric peaks merge. 
Table 1 Parameters used for the present studies, extracted from reference [1]

\begin{tabular}{lll}
\hline Parameters & Peak 1 (stage II) & Peak 2 (stage I) \\
\hline$\rho\left[\mathrm{g} \cdot \mathrm{cm}^{-3}\right]$ & 4.281 & 4.281 \\
$n_{L i}$ & 0.5 & 0.5 \\
$M\left[\mathrm{~g} \cdot \mathrm{mol}^{-1}\right]$ & 180.8 & 180.8 \\
$E_{1 / 2}[\mathrm{~V}]$ & 4.1336 & 3.9919 \\
$g_{1}$ & -0.81 & 1.13 \\
$g_{2}$ & 1.57 & -0.45 \\
$g_{3}$ & 9.58 & 2.34 \\
$D\left[\mathrm{~cm}^{2} \cdot \mathrm{s}^{-1}\right]$ & $1.69 \times 10^{-11}$ & $2.48 \times 10^{-11}$ \\
$k_{s}\left[\mathrm{~cm}^{-1}{ }^{-1}\right]$ & $3.07 \times 10^{-7}$ & $5.31 \times 10^{-7}$ \\
$\alpha$ & 0.502 & 0.506 \\
\hline
\end{tabular}

In the following discussion, we will denote the pair of peaks at the most positive potentials with " 1 " and the pair of peaks at the most negative potentials with " 2 ". Each pair is in turn separated into its oxidative $(\mathrm{O})$ and the reductive $(\mathrm{R})$ counterparts. So, the voltammetric peaks are denoted with $\mathrm{O} 1, \mathrm{R} 1, \mathrm{O} 2$ and R2, as illustrated in Figure $2 a$.

For a given particle size (same column in Figure 2) it is found that, as sweep rate increases, the peak potentials of $\mathrm{O} 1$ and $\mathrm{O} 2$ shift to higher values and the peak potentials for R1 and R2 shift to lower values. Concomitantly, it is found that all current peaks become flatter and broader as sweep rate increases. This effect is much more emphasized in the case of the larger particle sizes. Relatively high sweep rates, up to $20 \mathrm{mV} . \mathrm{s}^{-1}$, can be used for the smallest size $(h=134 \mathrm{~nm})$, without losing the resolution of the anodic peaks into two components. On the opposite range of particle sizes, for $h=$ $1 \mu \mathrm{m}$, we observe that $1 \mathrm{mV} \cdot \mathrm{s}^{-1}$ is high enough to merge the two anodic peaks together. A comparison of the smallest (nanometric) thickness $h=134 \mathrm{~nm}$ (Figure 2a) with the largest (micrometric) size $h=1 \mu m$ (Figure $2 g$ ) shows that the peak potential shifts are noticeably higher for the micrometric scale, at the same sweep rate. While for the nanometric size all voltammograms look quite similar in Figure $2 a$, for the micrometric scale the voltammograms in Figure $2 g$ become remarkably broad and flat as sweep rate increases.
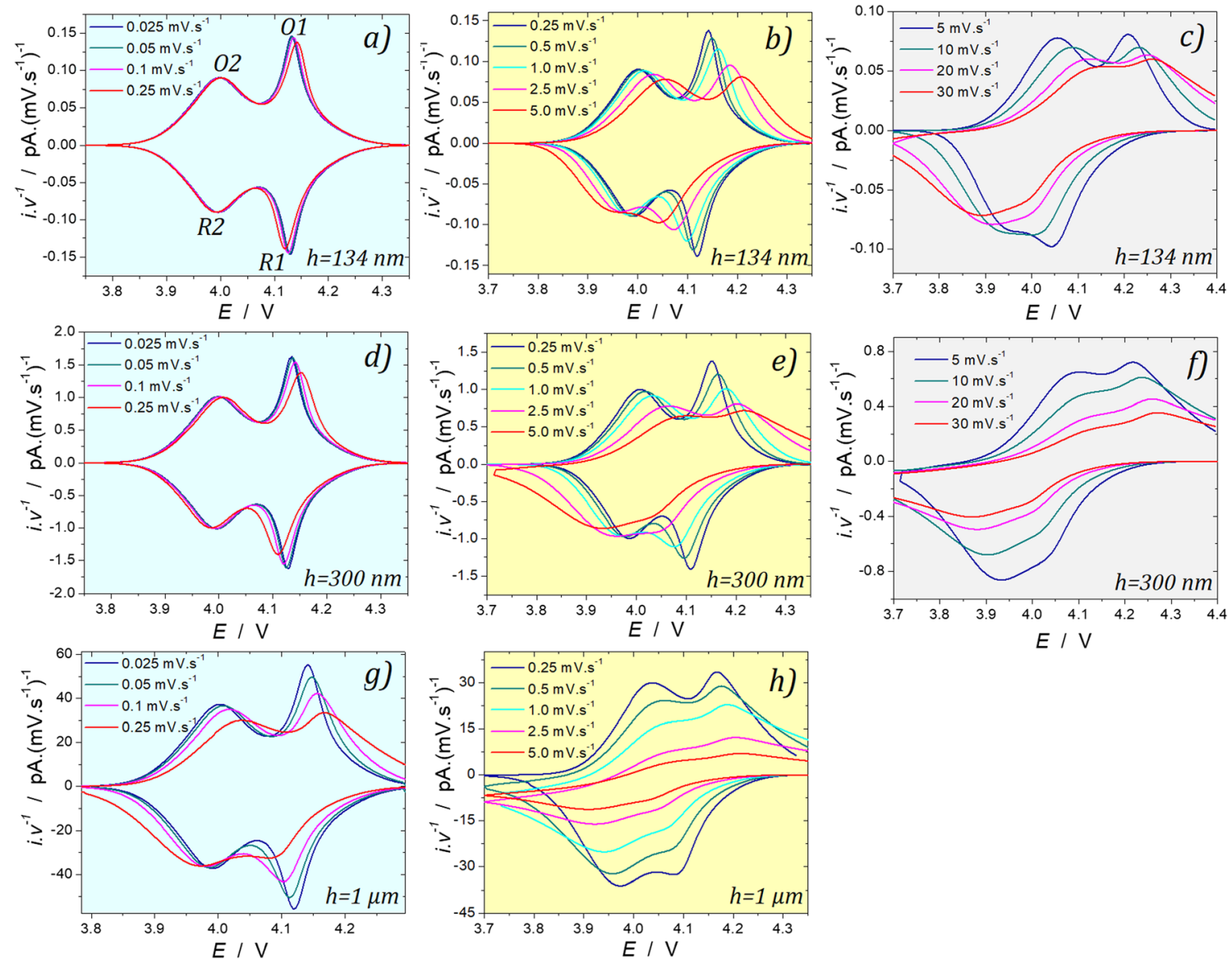

Fig. 2 Voltammograms for different sweep rates for planar geometry with $h=134 \mathrm{~nm}(a, b$ and $c), h=300 \mathrm{~nm}(d, e$ and $f)$ and $h=1 \mu \mathrm{m}(g$ and $h$ ). The current is normalized to the sweep rate. The sweep rates are indicated in each figure. The same set of sweep rates is placed in the same

column and shows the same background color (light blue, yellow or gray) to guide the eye. The voltammetric peaks are denoted with $\mathrm{O} 1, \mathrm{R} 1, \mathrm{O} 2$ and R2, as illustrated in Figure $2 a$ 
To perform a quantitative analysis with the data of Figure 2, let us denote with $E_{p o}$ the peak potential of the anodic peaks and with $E_{p r}$ the peak potential of the cathodic peaks. The $\left|E_{p o}-E_{p r}\right|$ differences for peaks 1 and 2 are presented in Figure $3 a$ as a function of sweep rate, for $h=134 \mathrm{~nm}$ (blue squares) and $h=1 \mu \mathrm{m}$ (red circles). The behavior of $\mid E_{p o}$ $-E_{p r} \mid$ vs sweep rate indicates that the thinner films are closer to equilibrium, for a given sweep rate. For thicker films, lower sweep rates are needed to decrease the $\left|E_{p o}-E_{p r}\right|$ peak potential difference.

To compare directly with experimental results, we address the voltammograms of Figure 4 from Mürter et al. [16], who performed voltammetric experiments for the present system. In this respect, we have simulated the voltammograms for $h=$ $300 \mathrm{~nm}$ (Figure $2 d-f$ ). In both cases, experiment and theory, the peak potential differences O1-R1 and O2-R2 are negligible at slow sweep rates (Figure $2 d$ ), denoting a quasiequilibrium state. These results suggest that the reversibility of the process is controlled by the diffusion length $h$. Then, for faster sweep rates (Figure $2 e$ and $f$ ), the peaks became broader and less defined.

The behavior of LMO spherical particles has been simulated in references [1-3, 29]. However, none of these theoretical works has systematically investigated the effect of nano-size on voltammetric measurements. We show here, in Figure $4 a$, current responses normalized by the volume, for spherical particles with different $h$ (particle size diameter $d=2 h$ ). All profiles were taken at the same sweep rate, $v=1 \mathrm{mV} . \mathrm{s}^{-1}$. As emphasized with black arrows, $\mathrm{O} 1$ and $\mathrm{O} 2$ shift towards positive potentials as particle size increases, while R1 and R2 shift to more negative potentials. As in the case of films, it appears that as $h$ becomes larger, the system moves away from equilibrium. This effect is clearly observed in Figure $4 b$, where $\left|E_{p o}-E_{p r}\right|$ is represented for several particle sizes, according

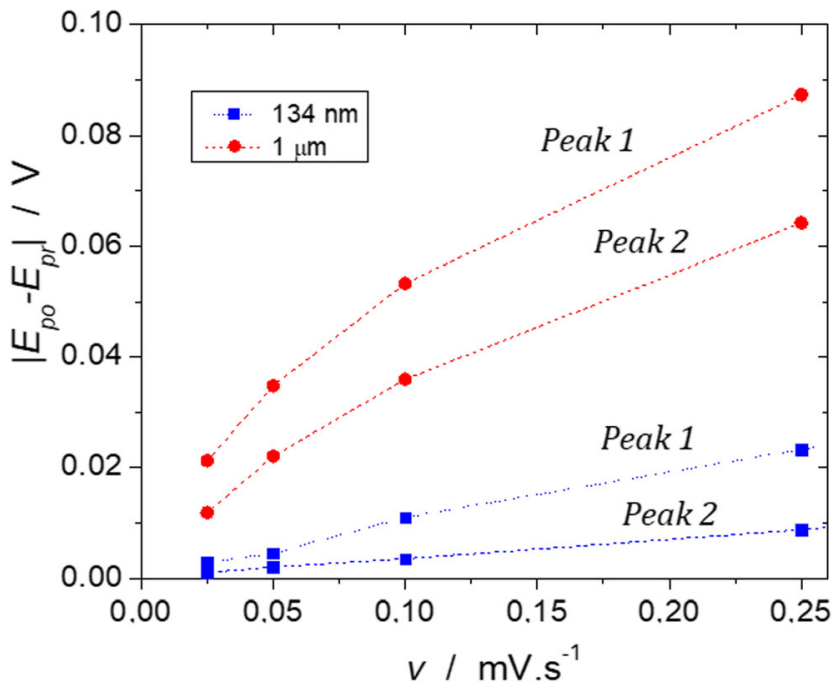

Fig. 3 Data analysis for the voltammograms of Figure 2. Peak potential difference for $h=134 \mathrm{~nm}$ and $h=1 \mu \mathrm{m}$ to the data of Figure $3 a$. It is remarkable how $\mid E_{p o}-$ $E_{p r}$ |increases with $h$, moving the system away from equilibrium, so that for larger particles, lower sweep rates are needed to decrease the peak potential difference. It is also observed, in Figure $4 a$, how the peaks become flatter and broader as system size increases, since diffusion becomes more important. This indicates that for nanometric particles, where diffusion plays a negligible role, very fast cyclic voltammograms can be run, as done by Tao et al. [15] with $\mathrm{LiMn}_{2} \mathrm{O}_{4}$.

The previous analysis shows that the key factor is the diffusion length $h$. However, here we are only taking into account the geometric and kinetic factors of isolated particles. In experimental systems, for isolated or composite electrodes, other effects must be considered, such as the preparation methods of the materials, particle agglomeration, the type of electrolyte or the diffusion times that the $\mathrm{Li}$ ions require to reach the particles $[6,36,37]$.

While several articles have dealt with the effect of LMO morphology on the electrochemical response of multiparticle systems [6, 38], the results of Tao et al. [15] have shown the sensitivity of the current response to the shape of a single particle. By changing $z$ in equation (1) and $j$ in equation (4), it is possible to simulate different particle shapes. With this purpose, we have considered the same $h=134 \mathrm{~nm}$ and the same volume $V=1.01 \times 10^{-14} \mathrm{~cm}^{3}$ for spherical, cylindrical and planar shapes of the electrode. Voltammetric results for the same $v=2 m V . s^{-1}$ are depicted in Figure $4 c$. It can be appreciated that each particle geometry has its characteristic current response, as Tao et al. have highlighted. Moreover, the results suggest that cylindrical and spherical geometries result in voltammograms closer to equilibrium, as compared with the planar geometry, since the peak potential differences $\mathrm{O} 1$ $\mathrm{R} 1$ and O2-R2 seem to be larger for the planar geometry as compared with the other two. It is worth noting this trend, going from spherical to cylindrical to planar, suggesting a systematic change with dimensionality of the host.

It is interesting to highlight the fact that the present results allow some conclusions to be drawn in line with those obtained from kMC simulations [17]: voltammetric peaks can be resolved at higher sweep rates with nanoparticles than those used with microparticles and the reductive peaks merge together at lower sweep rates than their oxidative counterpart.

The reason why the reductive peaks merge together at lower sweep rates as compared with their oxidative counterparts lies on the different diffusion coefficients for the processes taking place in voltammetric peaks, and can be understood as follows. A smaller diffusion coefficient produces a longer "tail" in the peak (slower drop of the current). Thus, in the sequence of peaks, the greater overlap between them will take place when the process with the slowest diffusion coefficient takes place first. In the present case, diffusion is slower for the more positive peak (peak 1): $D=1.69 \times 10^{-11} \mathrm{~cm}^{2} \cdot \mathrm{s}^{-1}$, as shown in Table 1. For peak 2, $D=2.48 \times 10^{-11} \mathrm{~cm}^{2} \cdot \mathrm{s}^{-1}$. 
Fig. 4 a) Simulated voltammograms for Li-ion insertion in LMO spherical particles of different sizes. The current is normalized by the volume of the sphere. The sweep rate was $v=1 m V \cdot s^{-1}$. The radii were: $25 \mathrm{~nm}, 50 \mathrm{~nm}, 125 \mathrm{~nm}$, $250 \mathrm{~nm}, 500 \mathrm{~nm}, 750 \mathrm{~nm}$ and $1000 \mathrm{~nm}(1 \mu \mathrm{m}) . b)$ Potential difference between anodic and cathodic peaks for LMO spherical particles of different sizes, denoted by the geometric factor $h$ (in this context, the sphere radius). c) Simulated voltammograms for LMO particles with spherical, cylindrical and planar geometries. The sweep rate was $v=2 \mathrm{mV} . \mathrm{s}^{-1}$, and all particles were given the same volume $1.01 \times 10^{-14} \mathrm{~cm}^{3}$
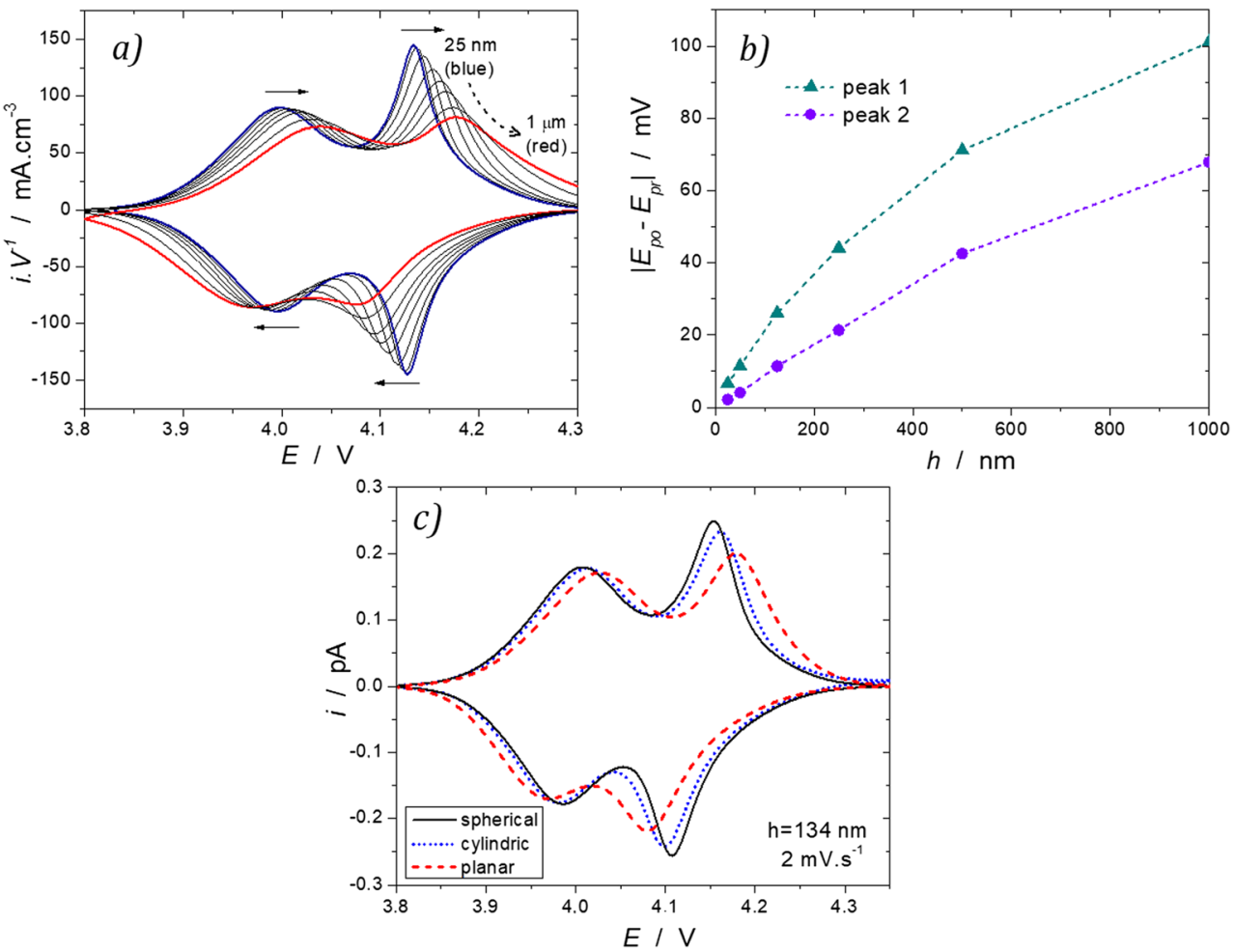

Thus, the largest overlap between voltammetric peaks should be observed in the reductive scan, as it is the case.

These results show the need to continue electrochemical studies with controlled geometry and particle sizes to properly understand $\mathrm{Li}$-ion intercalation into nano-sized materials.

\section{Conclusions}

Numerical simulations of voltammograms for Li-ion insertion in nanometric and micrometric systems were performed here, and the results were contrasted. Planar and spherical diffusion geometries were considered and the theoretical results were compared with suitable experimental data.

The present theoretical approximations show how particle size and geometry are crucial for electrochemical intercalation of $\mathrm{Li}^{+}$in electrode materials. Nanosized particles present better resolved voltammograms at higher sweep rates, as compared with microsized ones. The key factor for further control of the reversibility (and thus, a closer approach to equilibrium) of the system appears to be diffusion length. Thus, from this viewpoint, nanosized materials present better characteristics for application as electrodes for lithium-ion batteries. The application of the framework of Aoki et al. to $\mathrm{LiMn}_{2} \mathrm{O}_{4}$, indicates how the behavior of the system is sensitive to system size and electrochemical parameters. In this way, it appears as mandatory to construct a similar domain plot with a model considering interactions between inserted particles and the particle shape.

Although the decrease of particle size appears as appealing from the view point of the present simulations, there are a few questions concerning particle size that must be highlighted. The increase in the surface/volume ratio by decreasing particle size may lead to increasing electrolyte decomposition and passive growth formation, on the basis of surface catalytic activity [39]. This is specially a problem in the case of anode materials and could represent a problem for industry because it means a loss of electrolyte upon cell functioning. Besides, the crystallinity of the nano-sized particles is highly dependent on the synthesis method. If crystallinity is poor, reversibility complications arise [13].

Finally, it is emphasized that the control of shape and size in the synthesis of the particles for battery electrodes is an important factor, crucial for the performance of storage devices. Complementary efforts between experimental and theoretical studies are needed to improve the properties of batteries.

Acknowledgments E.P.M. Leiva acknowledges grants PIP CONICET $11220150100624 C O$, PUE/2017 CONICET, FONCYT PICT-20151605 and SECyT of the Universidad Nacional de Córdoba. Support by CCAD-UNC and GPGPU Computing Group, Y-TEC and an IPAC grant from SNCAD-MinCyT, Argentina, are also gratefully acknowledged. M.P. Mercer and H.E. Hoster thank the Faraday Institution (faraday.ac. uk; EP/S003053/1), grant number FIRG003, for funding. 


\section{References}

1. Vassiliev S, Levin EE, Nikitina VA (2016) Kinetic analysis of lithium intercalating systems : cyclic voltammetry. Electrochim Acta 190:1087-1099. https://doi.org/10.1016/j.electacta.2015.12. 172

2. A.K. Hjelm, G. Lindbergh, A. Lundqvist, Investigation of LiMn2O4 cathodes for use in rechargeable lithium batteries by linear sweep voltammetry - Part I. Theoretical study, J. Electroanal. Chem. 506 (2001) 82-91. doi:https://doi.org/10. 1016/S0022-0728(01)00512-5

3. Zhang D, Popov BN, White RE (2000) Modeling Lithium intercalation of a single spinel particle under Potentiodynamic control. J Electrochem Soc 147(3):831. https://doi.org/10.1149/1.1393279

4. Das SR, Majumder SB, Katiyar RS (2005) Kinetic analysis of the $\mathrm{Li}+$ ion intercalation behavior of solution derived nano-crystalline lithium manganate thin films. J Power Sources 139(1-2):261-268. https://doi.org/10.1016/j.jpowsour.2004.06.056

5. Wunde F, Nowak S, Mürter J, Hadjixenophontos E, Berkemeier F, Schmitz G (2017) Ion transport and phase transformation in thin film intercalation electrodes. Int J Mater Res 108:984-998. https:// doi.org/10.3139/146.111549

6. Xiao L, Guo Y, Qu D, Deng B, Liu H, Tang D (2013) Influence of particle sizes and morphologies on the electrochemical performances of spinel LiMn2O4 cathode materials. J Power Sources 225:286-292. https://doi.org/10.1016/j.jpowsour.2012.10.070

7. Nakayama N, Nozawa T, Iriyama Y, Abe T, Ogumi Z, Kikuchi K (2007) Interfacial lithium-ion transfer at the LiMn2O4 thin film electrode/aqueous solution interface. J Power Sources 174(2): 695-700. https://doi.org/10.1016/j.jpowsour.2007.06.113

8. Chung MD, Seo JH, Zhang XC, Sastry AM (2011) Implementing realistic geometry and measured diffusion coefficients into single particle electrode modeling based on experiments with single LiMn2O4 spinel particles. J Electrochem Soc 158(4):A371. https://doi.org/10.1149/1.3549161

9. Dokko K, Mohamedi M, Umeda M, Uchida I (2003) Kinetic study of Li-ion extraction and insertion at LiMn2O4 single particle electrodes using potential step and impedance methods. J Electrochem Soc 150(4):A425. https://doi.org/10.1149/1.1556596

10. Tang SB, Lai MO, Lu L (2008) Study on Li+-ion diffusion in nano-crystalline LiMn2O4 thin film cathode grown by pulsed laser deposition using CV, EIS and PITT techniques. Mater Chem Phys 111(1):149-153. https://doi.org/10.1016/j.matchemphys.2008.03. 041

11. Zhou HM, Zhu YH, Li J, Sun WJ, Liu ZZ (2019) Electrochemical performance of A1 2 O 3 pre-coated spinel LiMn 2 O 4. Rare Metals 38(2):128-135. https://doi.org/10.1007/s12598-014-0418-9

12. Cheng F, Wang H, Zhu Z, Wang Y, Zhang T, Tao Z, Chen J (2011) Porous LiMn $2 \mathrm{O} 4$ nanorods with durable high-rate capability for rechargeable Li-ion batteries. Energy Environ Sci 4(9):3668-3675. https://doi.org/10.1039/c1ee01795k

13. Xia H, Luo Z, Xie J (2012) Nanostructured LiMn2O4 and their composites as high-performance cathodes for lithium-ion batteries. Prog Nat Sci Mater Int 22(6):572-584. https://doi.org/10.1016/j. pnsc.2012.11.014

14. Nie XJ, Xi XT, Yang Y, Ning QL, Guo JZ, Wang MY, Gu ZY, Wu XL (2019) Recycled LiMn2O4 from the spent lithium ion batteries as cathode material for sodium ion batteries: electrochemical properties, structural evolution and electrode kinetics. Electrochim Acta 320:134626. https://doi.org/10.1016/j.electacta.2019.134626

15. Tao B, Yule LC, Daviddi E, Bentley CL, Unwin PR (2019) Correlative electrochemical microscopy of Li-ion (De)intercalation at a series of individual $\mathrm{LiMn} 2 \mathrm{O} 4$ particles. Angew Chemie - Int Ed 58(14):4606-4611. https://doi.org/10. 1002/anie.201814505
16. Mürter J, Nowak S, Hadjixenophontos E, Joshi Y, Schmitz G (2018) Grain boundary transport in sputter-deposited nanometric thin films of lithium manganese oxide. Nano Energy 43:340-350. https://doi.org/10.1016/j.nanoen.2017.11.038

17. E.M. Gavilán-Arriazu, O.A. Pinto, B.A. López De Mishima, D.E. Barraco, O.A. Oviedo, E.P.M. Leiva, Kinetic Monte Carlo applied to the electrochemical study of the Li-ion graphite system, Electrochim. Acta. 331 (2020). doi:https://doi.org/10.1016/j. electacta.2019.135439

18. E.M. Gavilán-Arriazu, M.P. Mercer, O.A. Pinto, O.A. Oviedo, D.E. Barraco, H.E. Hoster, E.P.M. Leiva, Effect of temperature on the kinetics and thermodynamics of electrochemical insertion of Liions into a graphite electrode, J. Electrochem. Soc. 167 (2020) 013533. doi:http://jes.ecsdl.org/content/167/1/013533.abstract

19. Leiva EPM, Perassi E, Barraco D (2017) Shedding light on the entropy change found for the transition stage II $\rightarrow$ stage I of Li-ion storage in graphite. J Electrochem Soc 164(1):A6154-A6157. https://doi.org/10.1149/2.0231701jes

20. M. Otero, A. Sigal, E.M. Perassi, D. Barraco, E.P.M. Leiva, Statistical mechanical modeling of the transition Stage II $\rightarrow$ Stage I of Li-ion storage in graphite. A priori vs induced heterogeneity, Electrochim. Acta. 245 (2017) 569-574. doi:https://doi.org/10. 1016/j.electacta.2017.05.128

21. Mercer MP, Otero M, Ferrer-Huerta M, Sigal A, Barraco DE, Hoster HE, Leiva EPM (2019) Transitions of lithium occupation in graphite: a physically informed model in the dilute lithium occupation limit supported by electrochemical and thermodynamic measurements. Electrochim Acta 324:134774. https://doi.org/10.1016/ j.electacta.2019.134774

22. Gavilán Arriazu EM, López de Mishima BA, Oviedo OA, Leiva EPM, Pinto OA (2017) Criticality of the phase transition on stage two in a lattice-gas model of a graphite anode in a lithium-ion battery. Phys Chem Chem Phys 19(34):23138-23145. https://doi. org/10.1039/C7CP04253A

23. Gavilán-Arriazu EM, Pinto OA, de Mishima BAL, Leiva EPM, Oviedo OA (2018) Grand canonical Monte Carlo study of Li intercalation into graphite. J Electrochem Soc 165(10):A2019-A2025. https://doi.org/10.1149/2.1211809jes

24. Perassi EM, Leiva EPM (2016) A theoretical model to determine intercalation entropy and enthalpy: application to lithium/graphite. Electrochem Commun 65:48-52. https://doi.org/10.1016/j.elecom. 2016.02.003

25. Gavilán-Arriazu EM, Pinto OA, López de Mishima BA, Barraco DE, Oviedo OA, Leiva EPM (2018) The kinetic origin of the Daumas-Hérold model for the Li-ion/graphite intercalation system. Electrochem Commun 93:133-137. https://doi.org/10.1016/j. elecom.2018.07.004

26. Aoki K, Tokuda K, Matsuda H (1984) Theory of linear sweep voltammetry with finite diffusion space. Part I. J Electroanal Chem 160(1-2):33-45. https://doi.org/10.1016/S0022-0728(84) 80113-8

27. Aoki K, Tokuda K, Matsuda H (1984) Theory of linear sweep voltammetry with finite diffusion space. Part II. Totally irreversible and quasi-reversible cases. J Electroanal Chem 160(1-2):33-45

28. Guin SK, Ambolikar AS, Das S, Poswal AK (2020) Advantage of fractional-calculus based hybrid-theoretical-computationalexperimental approach for alternating current voltammetry, Electroanalysis. https://doi.org/10.1002/elan.201900552

29. Zeng Y, Albertus P, Klein R, Chaturvedi N, Kojic A, Bazant MZ, Christensen J (2013) Efficient conservative numerical schemes for 1D nonlinear spherical diffusion equations with applications in battery modeling. J Electrochem Soc 160(9):A1565-A1571. https:// doi.org/10.1149/2.102309jes

30. Britz D, Strutwolf J (2016) Digital Simulation in Electrochemistry. https://doi.org/10.1007/978-3-319-30292-8 
31. Mehrer H (2007) Diffusion in solids. Springer, Berlin, Heidelberg. https://doi.org/10.1007/978-3-540-71488-0

32. Levi M, Aurbach D (1999) Frumkin intercalation isotherm - a tool for the description of lithium insertion into host materials: a review. Electrochim Acta 45(1-2):167-185. http://www.sciencedirect.com/ science/article/pii/S0013468699002029

33. Mercer MP, Finnigan S, Kramer D, Richards D, Hoster HE (2017) The influence of point defects on the entropy profiles of Lithium ion battery cathodes: a lattice-gas Monte Carlo study. Electrochim Acta 241:141-152. https://doi.org/10.1016/j.electacta.2017.04.115

34. Schlueter S, Genieser R, Richards D, Hoster HE, Mercer MP (2018) Quantifying structure dependent responses in Li-ion cells with excess Li spinel cathodes: matching voltage and entropy profiles through mean field models. Phys Chem Chem Phys 20(33): 21417-21429. https://doi.org/10.1039/c8cp02989j

35. B.E. Conway, two-dimensional and quasi-two-dimensional isotherms for Li intercalation and UPD processes at surfaces, Electrochim. Acta. 38 (1993) 1249-1258. doi: https://doi.org/10. 1016/0013-4686(93)80055-5

36. A.K. Hjelm, G. Lindbergh, A. Lundqvist, Investigation of LiMn2O4 cathodes for use in rechargeable lithium batteries by linear sweep voltammetry - Part II. Experimental study using thin films, single particles and composite electrodes, J. Electroanal. Chem. 509 (2001) 139-147. doi:https://doi.org/10.1016/S0022$0728(01) 00512-5$

37. Tian L, Yuan A (2009) Electrochemical performance of nanostructured spinel LiMn2O4 in different aqueous electrolytes. J Power Sources 192(2):693-697. https://doi.org/10.1016/j.jpowsour.2009. 03.002

38. Tang W, Yang X, Liu Z, Kasaishi S, Ooi K (2002) Preparation of fine single crystals of spinel-type lithium manganese oxide by $\mathrm{LiCl}$ flux method for rechargeable lithium batteries. Part 1. LiMn2O4. J Mater Chem 12(10):2991-2997. https://doi.org/10.1039/b203200g

39. Geder J, Hoster HE, Jossen A, Garche J, Yu DYW (2014) Impact of active material surface area on thermal stability of $\mathrm{LiCoO} 2$ cathode. J Power Sources 257:286-292. https://doi.org/10.1016/j. jpowsour.2014.01.116

Publisher's note Springer Nature remains neutral with regard to jurisdictional claims in published maps and institutional affiliations. 